\title{
A context-aware, predictive and protective approach for wellness monitoring of cardiac patients
}

\author{
Abdur Rahim Mohamad Forkan ${ }^{1}$, Weichich $\mathrm{Hu}^{2}$ \\ ${ }^{1}$ School of Computer Science and IT, RMIT University, Melbourne, Victoria, Australia \\ ${ }^{2}$ Dept. of Biomedical Engineering, Chung Yuan Christian University, Chung Li, Taoyuan, Taiwan
}

\begin{abstract}
Cardiovascular diseases are major cause of deaths throughout the world. In this work, we develop a contextaware system for wellness monitoring of older adults who leave alone in home and suffers from cardiac disease. The focus here is the integration of social networking services with conventional remote monitoring services by utilizing a scalable cloud platforms. The goal here is to expand patient's social linkage by identifying similarity in his/her cardiac conditions. Here we build a cloud-oriented context-aware model that captures health parameters using modern fitbit device and ECG sensors. The raw data are sent the cloud platforms provided by Amazon Web Service (AWS) where data is converted to high level context. Using social networks this high level context information is send to patient's friends, family and doctors who are interested to know about his/her health condition. The interested parties get notified by Facebook when the contextaware system detects any changes. That is, using this platform a cardiac patient who live alone and need continuous monitoring is always get connected with virtual community by means of his/her health information. . This is a new model that utilizes the context data generated by wearable sensors to create interesting social networking services. The system is also designed to promote cardiac patients to interact with their community of interest using various context-aware social services. The results obtained for this innovative model show a new approach of wellness monitoring using social networks.
\end{abstract}

\section{Introduction}

Ambient assisted living (AAL) is a home healthcare infrastructure where a patient leave alone in home and monitored by different wearable sensors and ambient devices. Traditional healthcare solutions are limited in services such as emergency monitoring and few types physical treatment for chronic and cardiac illness[1]. These are inadequate to accomplish the need of a lonely patient.
Long term social disconnection creates more complicated disease like mental depression, anxiety and dementia. This necessitates making existing context-aware solutions [2] more robust to support social services such that the system can automatically predict the current situation of the patient, send up-to-date information to his/her family member automatically and interconnect him in a virtual community to reduce the mental pain of being isolated. Modern wearable sensors such as a fitbit device or an ECG sensor can capture data continuously about patient's health status. FitBit provides a bundled application which enables user to synchronize the fitness data to computers and mobile devices so that the users might be able to read a general report about his/her recent activities. However, patients and their family members do not care about these statistics, they actually care about their physical health and mental status. Therefore, they need another application service which is more intelligent to solve their problems.

The principle aim of this paper is to enable patients with various cardiac conditions [3] to stay connected with their family, friends and the community by leveraging advances in communication infrastructure using context-aware service frameworks that facilitate the delivery of a wide range of services using smart sensors and devices. The situational context generated from wearable sensors and ambient devices contains health information of the user $[4,5]$. In our model, such contexts are merged with user's personal interest and processed in cloud servers [6] provided by Amazon Web Service (aws) [7] to identify groups of users having same context profile (i.e. same cardiac disease such as Tachycardia). Then, using social networking services these groups are interconnected to a community where they can exchange information about their disease. This facilitates the delivery of services among the members a group or between a peer by means of social network. Our cloud-based platform eases the management of context having large variations from vast number of $\mathrm{AAL}$ systems and allows simple integration of different social networking services using simple interface $[2,3]$.

The rest of this paper is organized as follows: $\S$ II sum- 
maries related work in the area. The functional components of the proposed model is presented in $\S \mathrm{III}$. $\S \mathrm{IV}$ provides analysis of case studies, experimental evaluations and $\S \mathrm{V}$ concludes our work.

\section{Related Works}

Some of the recent works use mobile social networks to serve specific social services to certain group such as people with disabilities[8]. Integration of context from body sensor network and social network[9] is another popular research work for providing services like healthcare data sharing inside social network and medical data visualization[10]. Senseface[11] is a framework to augment user context awareness with the social networks domain which dynamically select services for a community having same interest. SocialFusion[12] is another framework where data from mobile, sensor and social network are combined to serve context driven social services like watching video streams.

However, these works preliminary targeted for few general disease. In our previous work we showed how we can detect cardiac abnormalities using context-aware services [4]. From the inspiration of previous work here we specifically target to include social networking features along with cardiac abnormality detection and send notification to patient's friends and family upon occurrence of any type of abnormalities.

\section{The Methodology}

Our generalized cloud-based context-aware service model is depicted in Fig 1. In conjunction with cardiac abnormalities detection (e.g. tachycardia, bradycardia) for health monitoring purpose, the architecture also supports social services using social network such as Facebook. The context manager is the main collaborator of the system which binds an AAL user to his personal community of interest, maintains the repository of contexts and services related to abnormalities detection, and detects appropriate social services for the user. The major system functionalities are described below.

\subsection{AAL System}

In our model, large number of AAL systems can work simultaneously. An AAL system has a target user(elderly person), body sensors network (ECG sensor and FitBit), ambient devices, software applications and a running server for collecting sensor data locally and forwarding them to the cloud. The ECG sensor and FitBit device continuously generate data about user activity, heart condition, sleep and wake up time, calories burned, environmental conditions and so on. The local server (a smart phone/Laptop/personal computer) is connected to a secured datastore hosted on a cloud server in AWS (Fig 1). The datastore contains information related to user profile such as his diseases, interests, friends and family details, important contacts and other personal data as like in conventional social networks. We consider that each AAL user of our model will carry a smart phone (running on Android/iOS/WindowsOS). All the popular social networking apps are installed in the phone. User can update or modify his personal information using a simple application running on his mobile device. The application also have features for self-assessment (2(a)) of the user which takes yes/no feedback about some of his mental parameter like depression, loneliness, fears etc. These contexts are not easily detectable using sensors. The raw data from sensors and devices, and the application data from user feedback are combined and transmitted to a cloud-based context generator for context processing.

\subsection{Context Generator}

Context generator contains distributed context providers for converting raw data to high level context by intelligent feature selection techniques. The generated contexts are combined in a single context model by context aggregator (a distributed process running on cloud server). Thus, at any given time each of the AAL system has a context model containing physical and mental status of the user.

\subsection{Context Manager}

Context Manager (CM) is the main part of our model. The Context manager contains the cloud repository of context histories. CM first checks the existence of user in different social network. If it does not find any it creates a profile of the new user in every popular social networks using initial personal profile.

The CM collects new context from the AAL system via Context Generator, services from Service Providers and social context from social networks. Using new incoming context and context history of an AAL user the Context Manager finds a list of people having similar interests, diseases, health conditions and so on. The user of the AAL system is notified about suggested list in his mobile device (using facebook friend suggestion API) and can connect with other users. In this way the one's personal social network grows inside our model and that patient is actively involved in a community.

When an AAL user is connected to a community of interest he can enjoy different group-based context-aware social services. When the CM finds context from different AAL users it clusters them to multiple groups based on their interconnectivity and context similarity (e.g. simiar cardiac conditions). 


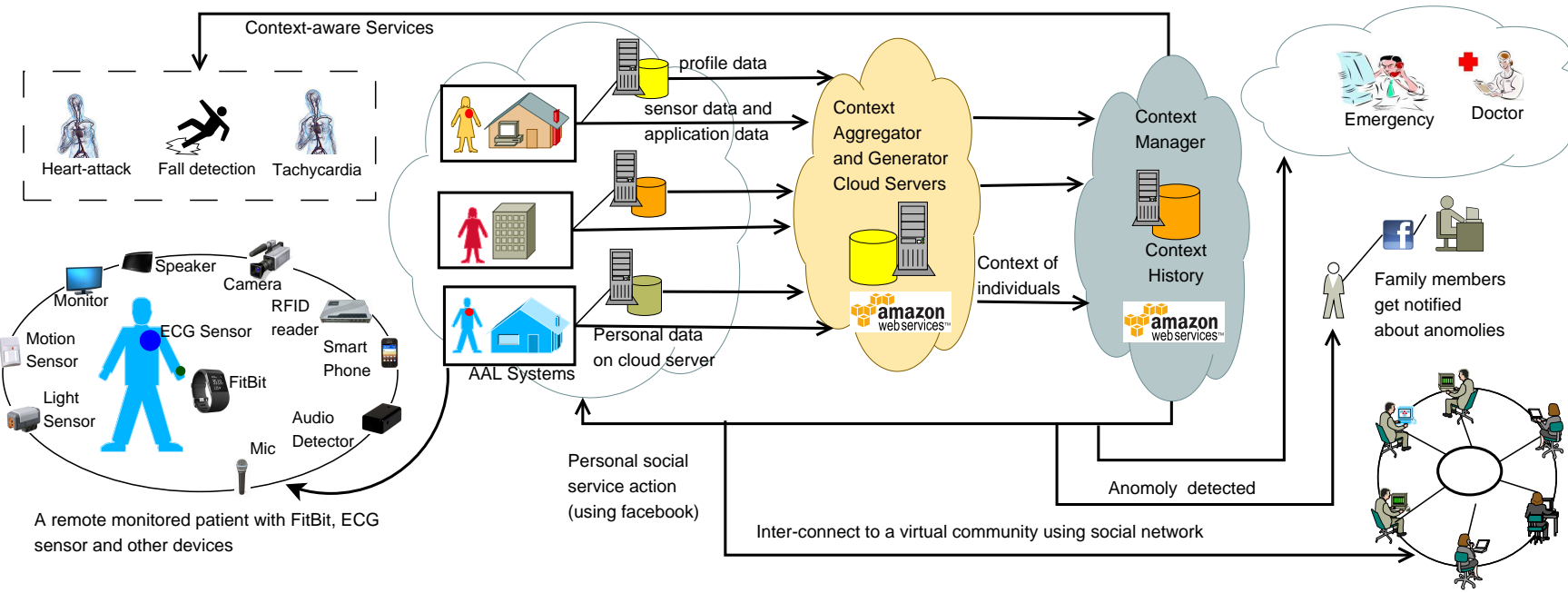

Figure 1. The Generic Cloud-based Model: Sensor data and application data generated in AAL systems converted to context in Context Aggregator cloud. Context Manager Cloud manages context from AAL systems, find abnormalities and notifies family members and doctors about patient's situation. The context-aware social cloud service also interconnects a list of patients having similar cardiac conditions using social network.

Once a group having similar context is identified the next step is to deliver the best possible social service to the group. Services are defined by rules containing specific values of context attributes. That is, services can also described by our standard context model. So, here the CM performs another similarity search. In this case the context model is compared with different services and a total score of matching is calculated. After that, CM picks the service with the best score value and delivers that service among the members of AAL systems as assisted service. As example, a facebook messenger conversation can be initiated to a group if similarity is found in some cardiac abnormalities.

The context manager is also capable of sending automatic alert notification using Facebook API. For example, if the CM detects that an AAL user is not taking his food properly from his activity context, it sends automated notification to his family members. The family member who view the notification can then contact with the suffered person using social network, sms, IM or phone. A sample example of notification interface is shown in Figure 2(b).

\section{Evaluation of Case Studies}

The proposed model is described completely from architectural point of view and we have not moved any real time implementation yet. However, we have begun evaluating various components of the system by simulating some case studies.

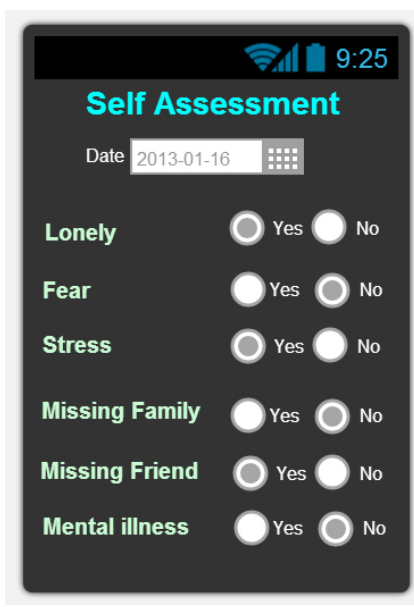

(a) Self-assessment appplication

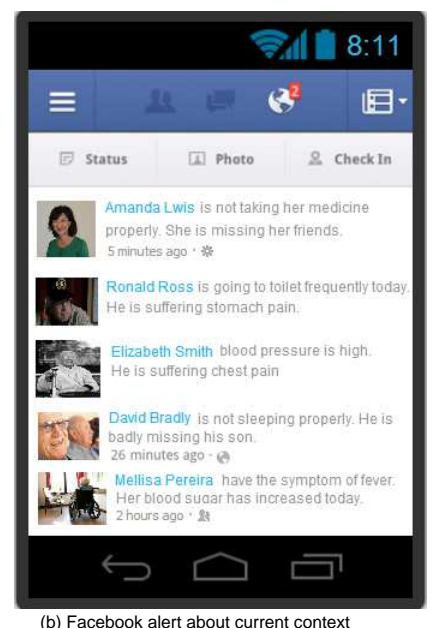

(b) Facebook alert about current context
Figure 2. Sample User Interface: (a) UI for selfassessment to identify mental context. (b) Example of social service using facebook feed update of an AAL user which is automatically generated by the system from observed context.

\subsection{Social Graph Generation}

We have created 100 samples of AAL system using our simulated environment and assigned some personal attributes (age, sex, friends and family contacts) to those samples. Each of the AAL system has a unique id and persistent information is kept in a MySQL database. We randomly assigned 12 different interest types and 8 different cardiac diseases in those samples. After that, we cluster the 
samples based on age, interest and disease. Thus, we got a social graph having 100 nodes and interconnected edges. From that graph, we can identify the social connectivity of an AAL user.

\subsection{Context-aware Social Service Selection}

To test context-aware services we created some Java class which represents the sensors and devices of AAL system. The member variables of each class have some numerical values which are used to classify high level context. After classification and aggregation we get 100 samples of context model (for 100 AAL systems). We did further clustering based on health condition contexts. Then we calculated the similarity values of the contexts and identified small clusters having higher context similarity. After that we applied service provisioning algorithm to find the best possible service from service database.

\subsection{Notifications using Social Network}

For simulating notification service, we have developed a test facebook application. The friend-list of each user is created using the social graph. When any anomaly is detected in a context sample of a test user we send notification to all people in his friend list.

\subsection{Performance Analysis}

So far we have evaluated the performance of our system by testing the time of social graph generation, context mapping, service selection and service delivery. We found that the whole process take few seconds in our simulated environment.

\section{Conclusion and Future Work}

In this paper, we presented a generalized context-aware framework for improving the social connectedness of socially disconnected patients people by social interactions in virtual communities. The proposed model facilitates the distributed nature of context-aware systems and web growth of social networking technology. By integrating diversified social networking features as context-aware service in AAL, our cloud-based model illustrates how selfsupporting people can contribute to the society actively. As part of ongoing work we will integrate data privacy and security feature in our model and will evaluate the performance of the model in real time.

\section{Acknowledgements}

The authors wish to acknowledge the support of RMIT University for funding the work presented in this paper.

\section{References}

[1] Sufi F, Khalil I, Mahmood A. Compressed ecg biometric: a fast, secured and efficient method for identification of cvd patient. Journal of medical systems 2011;35(6):1349-1358.

[2] Forkan ARM, Khalil I, Tari Z, Foufou S, Bouras A. A context-aware approach for long-term behavioural change detection and abnormality prediction in ambient assisted living. Pattern Recognition 2015;48(3):628-641.

[3] Ibaida A, Khalil I, Sufi F. Cardiac abnormalities detection from compressed ecg in wireless telemonitoring using principal components analysis (PCA). In 5th International Conference on Intelligent Sensors, Sensor Networks and Information Processing (ISSNIP), 2009. IEEE, 2009; 207-212.

[4] Forkan A, Khalil I, Tari Z. Context-aware cardiac monitoring for early detection of heart diseases. In Computing in Cardiology Conference (CinC), 2013. IEEE, 2013; 277 280.

[5] Forkan A, Khalil I, Tari Z. CoCaMAAL: A cloud-oriented context-aware middleware in ambient assisted living. Future Generation Computer Systems 2014;35:114-127.

[6] Forkan A, Khalil I, Ibaida A, Tari Z. BDCaM: Big data for context-aware monitoring-a personalized knowledge discovery framework for assisted healthcare. IEEE Transactions on Cloud Computing 2015;.

[7] Amazon Web Service. http://aws.amazon.com, 2014.

[8] Chang Y, Liu H, Wang T. Mobile social networks as quality of life technology for people with severe mental illness. Wireless Communications IEEE 2009;16(3):34-40.

[9] Domingo M. A context-aware service architecture for the integration of body sensor networks and social networks through the ip multimedia subsystem. Communications Magazine IEEE 2011;49(1):102-108.

[10] Bauschlicher D, Bauschlicher S, ElAarag H. Framework for the integration of body sensor networks and social networks to improve health awareness. In Proceedings of the 14th Communications and Networking Symposium. Society for Computer Simulation International, 2011; 19-26.

[11] Rahman M, El Saddik A, Gueaieb W. Augmenting context awareness by combining body sensor networks and social networks. Instrumentation and Measurement IEEE Transactions on $2011 ; 60(2): 345-353$.

[12] Beach A, Gartrell M, Xing X, Han R, Lv Q, Mishra S, Seada K. Fusing mobile, sensor, and social data to fully enable context-aware computing. In Proceedings of the Eleventh Workshop on Mobile Computing Systems \& Applications. ACM, 2010; 60-65.

Address for correspondence:

Abdur Rahim Mohammad Forkan

School of Computer Science and IT

RMIT University,

City Campus, 414-418 Swanston Street, Melbourne,

VIC-3001, Australia

abdur.forkan@rmit.edu.au 